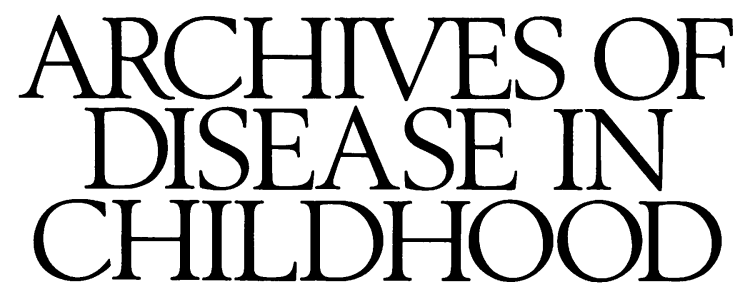

\title{
Assisted reproduction and its implications for paediatrics
}

The relative rarity of multiple birth has made its occurrence in our society a subject of keen interest, sometimes approaching awe. The overwhelming press coverage of the birth and subsequent progress of quintuplets and sextuplets attests to this, but now that these higher multiple births have become, in the eyes of the media, relatively common their newsworthy 'human interest' appeal has proportionately waned. The interest that many feel when hearing of four, five, or six babies born at one time may over-ride other very important considerations related to these births, notably their medical and social implications for the family as well as the hospital and community services. For paediatricians and all those involved with these families it is timely to consider the effects of assisted reproduction in terms of the increasing numbers of higher multiple births and the manner in which they impinge on our services.

From the late 1950s and early 1960s there was a worldwide decline in the number of twins. ${ }^{1}$ In the United States this decline was noted somewhat earlier and there was a similar proportionate reduction in the rate of triplet births from the 1930 s to the 1940 s. $^{2}$ These changes appear to be due to reduction in the dizygous twinning rates. In England and Wales (and probably other developed countries) there was an increase in the proportion of triplets and other higher multiple births in the late 1970s. ${ }^{3}$ Data from the Office of Population Censuses and Surveys (OPCS) show that in the 15 year period from 1956 to 1970 there were 19 sets of higher multiple births (quadruplets, quintuplets, and sextuplets) and in a similar period from 1971 to 1985 there were 78 sets, a fourfold increase. ${ }^{3}$ The increase in the numbers of higher multiple births is certainly due to the introduction of methods for treating the infertile couple and these include ovarian stimulation, gamete intrafallopian transfer (GIFT), and in vitro fertilisation (IVF). Unfortunately, it is neither clear what proportion of triplets and higher multiple births is due to these methods of assisted reproduction, nor the relative importance of each of these techniques to the total number of multiple births.

\section{Assisted reproduction}

Infertility may be due to many causes including anovulation, fallopian tube blockage, ovarian underdevelopment, abnormalities of the sperm, and a group where no cause can be found. There is a range of disorders associated with anovulation and drug regimens are available to stimulate the production of ova which are fertilised in the fallopian tube after normal sexual intercourse. Clomiphene citrate is the most widely used drug for ovarian stimulation. Its use alone is often successful in treating the anovulatory woman. Gonadotrophin treatment (either human chorionic gonadotrophin or human menopausal gonadotrophin) may be used in association with clomiphene to trigger ovulation. Hyperstimulation of the ovaries may cause multiple ova to be produced with the risk of multiple birth. Clomiphene alone rarely causes multiple ovulation and the multiple pregnancy rate is in the order of $5 \%$.The risk of multiple pregnancy (twins or more) after gonadotrophin use is considerably higher-in the order of $15-45 \%$ and for triplets $5-6 \%{ }^{4}$

GIFT is a new technique, first used successfully in 1984. It appears to be useful in the further management of unexplained infertility. Multiple ova are first harvested laparoscopically after ovarian stimulation. A number of ova are selected for replacement and inserted into the fallopian tube under laparoscopic control together with approximately $10^{6}$ spermatozoa. Success rates for pregnancy are reported to be $25-40 \%$ and it is becoming a more widely used technique due to its requirements for relative low technology and low cost. The risk of multiple birth is $19-38 \% .5^{5}$

IVF was initially developed in order to treat the woman with occluded or absent fallopian tubes, but is now more widely used for treating other causes of infertility. The procedure involves harvesting ova after ovarian stimulation as described above. They are then fertilised by spermatozoa outside the body and cultured for a short time. Great care must be taken to maintain the culture environment to ensure normal development during the period of incubation. Fertilised oocytes are carefully inspected and if suitable are replaced in the culture medium until they are inserted into the uterus transcervically at approximately 48 hours from insemination. Unused fertilised ova (now referred to as pre-embryos) may be frozen for insertion at later cycles. The pregnancy success rate for each patient is in the order of $10-15 \%$, and the rate of multiple birth is $22 \%{ }^{6}{ }^{7} \mathrm{~A}$ recent Medical Research Council report on IVF and GIFT pregnancies indicate that $80 \%$ of all such pregnancies occurred after treatment in just three centres; all of which operated outside the National Health Service. ${ }^{6}$

Complications of triplets and higher multiple births The risk of fetal, neonatal, and infant death is considerably increased in triplets and higher multiple births. In the years 1975-83 the perinatal mortality and infant mortality rates 
for triplets were $164 \cdot 5$ and $147 \cdot 7$ respectively (England and Wales) compared with mortality rates of 13.8 and 11.7 for singleton births over the same period. ${ }^{3}$ For quadruplets the mortality rate was between $40-50 \%$ higher than that of triplets. The mortality rates are increased for a number of reasons. These include uteroplacental compromise, premature delivery, and congenital abnormality.

Maternal complications of triplet and higher multiple births include pregnancy induced hypertension, anaemia, and antepartum or postpartum haemorrhage. Routine admission of these women for bed rest is recommended by many obstetricians in the latter part of the second trimester; a practice that is both expensive and disruptive to existing children in the family. In addition, obstetricians may recommend the routine use of tocolytics (usually betamimetics) and regular injections of corticosteroids to the pregnant women in order to avoid excessive uterine activity and enhance fetal lung maturity. Endocervical cerclage has been advocated as a routine procedure in the management of triplets and quadruplets, but the efficacy of this form of management has not been proved. Other fetal risks include placental complications such as abruption, intrauterine growth retardation secondary to placental insufficiency, and malpresentation.

Prematurity is the single most important cause for the increased neonatal mortality in higher multiple births. The incidence of prematurity in triplets is reported to be $88 \%,{ }^{8}$ and the mean gestational age at which triplets and quadruplets were delivered was 34-35 weeks. ${ }^{89}$ Despite the surprising degree of maturity in triplets and higher multiples there is a significant number who are born extremely premature and who develop major neonatal complications. It is of some interest that singleton infants born after IVF treatment showed a considerably higher risk of premature delivery than would be expected in a population of naturally occurring pregnancies. ${ }^{67}$ It is possible that the factors determining infertility are also an additional risk factor for prematurity.

Despite an initial suggestion that infants born as the result of GIFT and IVF were more likely to have congenital abnormality, ${ }^{10}$ this has not been confirmed in later studies. Monozygotic twins have a significant risk of cerebral injury, particularly if one twin dies in utero. ${ }^{11}$ There is evidence that there is an increased risk of monozygotic twins after the use of ovarian stimulation ${ }^{12}$ and IVF. ${ }^{13}$ There are very few data on the developmental outcome of the survivors of higher multiple births. A recent study from a tertiary referral centre in Toronto reported follow up data in 84 infants from 29 triplet, quadruplet, and quintuplet pregnancies and found that only one child had a major handicap. ${ }^{14}$ Prospectively ascertained cohorts of these infants should be followed up on a geographical basis in order to establish further the quality of life in these high risk children.

Until recently the social aspects of higher multiple births have been largely neglected. The major problems facing these families once the children are home are lack of space and the need for extra help to meet the needs of all the children. The parents of triplets found that their greatest problem was lack of sleep and the unexpected strain on the family finances. ${ }^{15}$

In an effort to understand further the problems associated with grand multiple births, a retrospective study of 'triplet and higher order births' was undertaken which attempted to ascertain all cases over a five year period in the early 1980 s. These results have just been published in book form. ${ }^{16}$ Both medical and social problems are discussed, but it is the family problems that are most startling. One mother of 6 month old triplets reported that she had calculated that just caring for her babies together with household chores took up 197.5 hours a week, yet there are only 168 hours in each week! Despite this, almost $40 \%$ of families with triplets receive no home help from their local authority.

\section{The control of higher multiple births}

In view of the apparent considerable increase in the numbers of higher multiple pregnancies, and because these fetuses and babies are at greater risk of death, it is important to consider ways of controlling the number of such deliveries. There are two approaches: applying controls to the assisted reproduction techniques and selective termination of some members of the multiple fetal group. This latter process has been called selective fetocide.

The recent Human Embryology and Assisted Reproduction Act seeks to control centres involved in IVF and carefully limit the numbers of embryos that are replaced to three. This still makes the possibility of triplets likely and some of the embryos may yet divide to monozygotic twins thereby producing quadruplets or possibly more conceptuses. GIFT has been excluded from the legislation and it appears to be an increasingly common form of treatment. Multiple ova (well in excess of three) are inserted in some centres. ${ }^{5}$ In my view GIFT should also be more carefully regulated and confined to licensed centres. The major contribution towards the birth of higher multiple births is likely to be ovarian stimulation. This procedure is undertaken in many district general hospitals, possibly without appropriate biochemical or ultrasound control. A strong case can be made for requiring the management of infertility to be confined to subregional centres which are suitably equipped to undertake these procedures in as controlled a manner as possible.

A more controversial approach to the inevitable occurrence of higher multiple pregnancy is termination of selected fetuses, thereby reducing the number from four, five, or six down to one or two. There are now many descriptions in the literature of these techniques. The safest appears to be instillation of potassium chloride directly into the fetal heart through a transabdominal percutaneous needle. This is most successful when performed in the first trimester of pregnancy. ${ }^{17}$ Unfortunately this procedure may cause all the fetuses to be lost as the result of premature labour; a particular tragedy for a woman who had been treated initially for infertility. In Britain the legal position of this technique is unclear. It has been suggested that because selective reduction does not cause 'termination of pregnancy', it contravenes the Abortion Act and may subject the operator to prosecution. ${ }^{18}$ The American and European experience suggests that selective reduction of multiple pregnancy is a valuable technique in some cases.

\section{The future}

There is no reason to believe that there will be a reduction in the steep rise in the numbers of higher multiple births and this continues to cause anxiety to those providing intensive care facilities for premature infants. It is clearly important that the numbers of triplets and higher multiple births continue to be closely monitored. We must also determine the exact contribution that methods of assisted reproduction make to these numbers. In 1989, the British Association of Perinatal Medicine together with the British Paediatric Surveillance Unit surveyed all cases of triplets and higher multiple births delivering after 20 weeks of gestation in Britain in order to determine the proportion due to ovarian stimulation and other techniques. These results will be available soon.

We must establish a dialogue with our gynaecological colleagues who are involved in the treatment of the infertile couple in order to coordinate our efforts. This is particularly 
important in those centres that provide services outside the National Health Service as some of these higher multiple births will require intensive care in inadequately funded health service cots. Perhaps a start could be made if there were agreement that an insurance policy should be taken out by those involved in these forms of treatment to cover the cost of neonatal intensive care should this be required as the result of a higher multiple birth.

MALCOLM I LEVENE

University Department of

Paediatrics and Child Health,

D Floor, Clarendon Wing,

Leeds General Infirmary,

Belmont Grove,

Leeds LS2 9NS

1 Anonymous. Worldwide decline in dizygotic twinning. [Editorial.] $B M F$ 1976;i:1553.

2 Guttmacher AF. The incidence of multiple births in man and some of the other unipara. Obstet Gynecol 1953;2:22-35.

3 Botting BJ, MacDonald Davies I, MacFarlane AJ. Recent trends in the incidence of multiple births and associated mortality. Arch Dis Child 1987;62:941-50.
4 Scialli AR. The reproductive toxicity of ovulation induction. Fertil Steril 1986;45:315-23.

5 Craft I, Ah-Moye M, Al-Shawaf T, et al. Analysis of 1071 GIFT proceduresthe case for a flexible approach to treatment. Lancet 1988;i:1094-8.

6 MRC Working Party on Children Conceived by In Vitro Fertilisation. Births in Great Britain resulting from assisted conception, 1978-87. BMF in Great Britain

7 Australian In-Vitro Fertilization Collaborative Group. In-vitro fetilization pregnancies in Australia and New Zealand, 1979-1985. Med f Aust 1988;148:429-36.

8 Newman RB, Hamer C, Miller MC. Outpatient triplet management: a contemporary review. Am $\mathcal{F}$ Obstet. Gynecol 1989;161:547-55.

9 Ron-el R, Caspi E, Schreyer P, Weinraub Z, Arieli S, Goldberg MD. Triplet and quadruplet pregnancies and management. Obstet Gynecol 1981;57:45863.

10 Lancaster PAL. Congenital malformations after in-vitro fertilisation. Lancet 1987;ii:1392-3.

11 Larroche J-C, Droulle P, Delezoide AL, Narcy F, Nessmann C. Brain damage in monozygous twins. Biol Neonate 1990;57:261-78.

12 Derom C, Vlietinck R, Derom R, Van den Berghe $H$, Thiery M. Increased monozygotic twinning rate after ovulation induction. Lancet 1987;i:1236-8.

13 Edwards R, Mettler L, Walters D. Identical twins and in vitro fertilisation. f In Vitro Fert Embryo Transfer 1986;3:114-7.

14 Gonen R, Heyman E, Asztalos EV, et al. The outcome of triplet, quadruplet, and quintuplet pregnancies managed in a perinatal unit: obstetric, neonatal, and quintuplet pregnancies managed in a perinatal unit: obstet

15 Berg G, Finnstrom O, Selbring A. Triplet pregnancies in Linkoping, Sweden, 1973-1981. Acta Genet Med Gemellol (Roma) 1983;32:251-6.

16 Botting BJ, Macfarlane AJ, Price FV, eds. Three, four and more. A study of triplet and higher order births. London: HMSO, 1990

17 Berkowitz RL, Lynch L, Chitkara U, Wilkins IA, Mehalek KE, Alvarez E. Selective reduction of multifetal pregnancies in the first trimester. $N$ Englf Med 1988;318:1043-7.

18 Howie PW. Selective reduction in multiple pregnancy. BMF 1988;297: 433-4.

\section{Development of intestinal motility}

Normal intestinal motility is essential for the orderly movement of nutrients from one specialised part of the gastrointestinal tract to the next. Disturbances will result in abdominal distension and vomiting when the transit of luminal contents slows down, and in diarrhoea when it speeds up. Under both circumstances the normal digestion and absorption of nutrients and the excretion of waste products will be compromised. In preterm infants, immature intestinal motility is one of the main causes of the 'physiological ileus' that often occurs. ${ }^{1}$

\section{Control of intestinal motility}

Patterns of normal intestinal motor activity have been described for most sections of the gastrointestinal tract and it is clear that a hierarchy of controls is responsible for determining the contractile behaviour of the gut. ${ }^{2}$ At the lowest level the smooth muscle cells exhibit spontaneous rhythmic electrical activity which determines the timing and frequency of contractile activity. This is further modulated by the action of the enteric nervous system and by the influences of a range of humoral substances that are loosely referred to as 'gut hormones'. The enteric nervous system is essential for the coordination of motor activity, while the gut hormones modulate the tone and excitability of the smooth muscle cells. The sympathetic and parasympathetic limbs of the autonomic nervous system link the enteric nervous system to the nuclei of the brain stem and to higher levels within the central nervous system. The enteric nervous system is complex, containing a similar number of neurones to the spinal cord, and although central connections exist one should think of the enteric nervous system as a autonomous unit that is modulated rather than controlled by the central nervous system.

The cells that ultimately form the enteric nerves migrate from the neural crest during the first trimester, and after colonisation of the gut they undergo a prolonged period of maturation that is in part determined by the microenvironment in which they find themselves. ${ }^{3}$ Evidence from animal studies suggests that these enteric neurones remain 'plastic' and open to environmental influences until a relatively late stage in their development. ${ }^{4}$ Histological and ultrastructural studies show the presence of enteric nerves and smooth muscle cells within the wall of the human intestine during the second trimester, and by 22 weeks' gestation the small intestine is 'histologically mature'. ${ }^{5}$ From clinical experience, however, we know that the intestines of preterm infants are often intolerant of enteral feeds, indicating that immaturity in the development of motor control is often a limiting factor in our ability to feed by the enteral route. ${ }^{1}$. Our present understanding of the development of these controls is outlined below.

\section{Sucking and swallowing}

The actions of sucking and swallowing a feed rely on the integration of control between a larger number of cranial nerve nuclei and are clearly dependent on the maturation of brain stem function. Swallowing has been observed in utero as early as 16 weeks' gestation and from studies using red blood cells tagged with ${ }^{51} \mathrm{Cr}$ injected into the amniotic fluid we know that the volume of fluid swallowed increases from $13 \mathrm{ml} /$ day at 20 weeks to $450 \mathrm{ml} /$ day at full term. ${ }^{6}$ It must be remembered, however, that the fetus is immersed in a bath of amniotic fluid and does not have to coordinate swallowing with either sucking or the protection of the airway. In preterm infants sucking movements are generally feeble but by 34-35 weeks' gestation a mature nutritive sucking pattern has developed. 78

Gryboski was the first to outline the normal patterns of oesophageal motility in both term and preterm infants. ${ }^{9}$ In adults a swallow initiates relaxation of the upper oesophageal spincter so that food can be carried along the length of the oesophagus by a primary peristaltic wave to the lower sphincter, which then relaxes to allow its passage into the stomach. ${ }^{10}$ While this pattern is present in full term infants who suck effectively, the motility of the oesophageal body in preterm infants is characterised by poorly propagated, low 